Annuaire suisse de politique de développement

12 | 1993

Annuaire Suisse - Tiers Monde 1993

\title{
Partenaires dans la recherche avec les pays en développement
}

Forschungs-Partnerschaft mit Entwicklungsländern

Thierry A. Freyvogel

\section{CpenEdition}

1 Journals

Édition électronique

URL : http://journals.openedition.org/aspd/1430

DOI : $10.4000 /$ aspd. 1430

ISSN : 1663-9669

Éditeur

Institut de hautes études internationales et du développement

Édition imprimée

Date de publication : 1 février 1993

Pagination : 215-224

ISSN : 1660-5934

\section{Référence électronique}

Thierry A. Freyvogel, «Partenaires dans la recherche avec les pays en développement », Annuaire suisse de politique de développement [En ligne], 12 | 1993, mis en ligne le 30 avril 2013, consulté le 08 septembre 2020. URL : http://journals.openedition.org/aspd/1430 ; DOI : https://doi.org/10.4000/ aspd. 1430

Ce document a été généré automatiquement le 8 septembre 2020

(c) The Graduate Institute I Geneva 


\section{Partenaires dans la recherche avec les pays en développement}

Forschungs-Partnerschaft mit Entwicklungsländern

Thierry A. Freyvogel

\section{NOTE DE L'ÉDITEUR}

En français, résumé seulement. Lire l'article original en allemand dans Schweizerisches Jahrbuch für Entwicklungspolitik : « Forschungs-Partnerschaft mit Entwicklungsländern ", http://sjep.revues.org/1248.

\section{RÉSUMÉS}

Dans un grand nombre de pays en développement, il n'existe toujours pas de réelles capacités de recherche, en dépit des initiatives de toute nature prises au cours des dernières décennies. Or, dans la perspective du développement, et pour résoudre les problèmes planétaires cruciaux qui sont les nôtres, ces capacités de recherche sont indispensables. A l'heure actuelle, la situation n'est propice ni dans les pays en développement, ni dans les pays industriels. Dans les premiers, il n'existe souvent pas de politique durable et cohérente de la recherche et, par voie de conséquence, il n'y a pas non plus de débouchés professionnels convenables pour les rares chercheurs indigènes; dans les seconds, la communauté scientifique marque peu d'intérêt pour les projets de recherche axés sur le développement et réalisés au profit des pays en développement. Par ailleurs, la capacité de travailler de manière interdisciplinaire fait défaut partout.

Pays industriel parmi d'autres, la Suisse voudrait apporter sa pierre à la mise en place du 
potentiel de recherche dont les pays en développement ont besoin. Pour ce faire, elle doit se doter d'une conception, en d'autres termes d'une «stratégie ». Sans doute les actions menées par la Suisse en faveur de la recherche dans les pays en développement ont-elles parfois produit des résultats remarquables. Elles ont cependant été réalisées sans but concerté, en ordre dispersé et souvent sans aucun suivi; par conséquent, elles n'ont en général pas eu d'effet durable. La stratégie en préparation doit donc consister d'abord à évaluer le travail accompli et, le cas échéant, à le poursuivre après en avoir amélioré les modalités ; elle doit cependant viser à mieux coordonner les travaux et à les mettre à exécution de manière concertée. Cette stratégie comprendra de plus un programme spécial à long terme. Celui-ci devrait porter sur l'utilisation des ressources naturelles, être réalisé entre partenaires suisses et des pays en développement intéressés au projet. De la sorte, il est probable que bon nombre d'obstacles majeurs qui entravent une recherche indépendante dans les pays en développement pourraient être surmontés. 